\title{
Novel Water Overlayer Growth on Pd(111) Characterized with Scanning Tunneling Microscopy and Density Functional Theory
}

\author{
J. Cerdá, ${ }^{1}$ A. Michaelides, ${ }^{2,3}$ M.-L. Bocquet, ${ }^{3, *}$ Peter J. Feibelman, ${ }^{4}$ T. Mitsui, ${ }^{5}$ M. Rose, ${ }^{5}$ E. Fomin, ${ }^{5}$ and M. Salmeron ${ }^{5}$ \\ ${ }^{1}$ Instituto de Ciencia de Materiales de Madrid, CSIC, Cantoblanco, 28049 Madrid, Spain \\ ${ }^{2}$ Department of Chemistry, University of Cambridge, Lensfield Road, Cambridge CB2 1EW, U.K. \\ ${ }^{3}$ Laboratoire de Chimie, UMR 5182, Ecole Normale Supérieure, Lyon, France \\ ${ }^{4}$ Sandia National Laboratories, Albuquerque, New Mexico 87185-1415, USA \\ ${ }^{5}$ Lawrence Berkeley Laboratory, University of California, Berkeley, USA
}

(Received 6 May 2004; published 7 September 2004)

\begin{abstract}
Scanning tunneling microscopy (STM) images of water submonolayers on $\mathrm{Pd}(111)$ reveal quasiperiodic and isolated adclusters with internal structure that would ordinarily be ascribed to icelike puckered hexagonal units. However, density functional theory and STM simulations contradict this conventional picture, showing instead that the water adlayers are composed mainly of flat-lying molecules arranged in planar water hexagons. A new rule for two dimensional (2D) water growth is offered that generates the structures observed experimentally from planar hexamer units.
\end{abstract}

DOI: 10.1103/PhysRevLett.93.116101

It is hard to overstate the importance of water-solid interactions. They play a key role in many areas of science, e.g., heterogeneous catalysis and corrosion. Nonetheless, understanding water-solid interactions has not proven easy. Because water is a closed-shell molecule, it interacts relatively weakly with its surroundings. For this reason, quantitative measurement of the structure of water adsorbed on surfaces is a delicate matter, and little detailed structural information is available in the hundreds of papers published on water-solid interactions [1].

The conventional picture of wetting layers on closepacked metal surfaces is that the water molecules form a "puckered hexagonal bilayer," residing in two distinct orientations and at two heights above the outer metal layer, in an adsorbed, H-bonded network [1]. The lower water molecules, lying nearly flat, contribute two donor $\mathrm{H}$ bonds to the hexagonal network while their $\mathrm{O}$ atoms attach to the metal via lone-pair hybridization with the $d$ band. The higher-lying molecules, by contrast, provide one donor $\mathrm{H}$-bond to the network, and have one $\mathrm{O}-\mathrm{H}$ free or dangling out of plane. If the free $\mathrm{H}$ atoms lie above the oxygens to which they are bonded, as they should if tetrahedral bonding is preferred, then the adsorption arrangement is an "H-up" bilayer. If the free $\mathrm{OHs}$ are inverted, as shown by recent studies to be possible [2], then the adlayer is referred to as "H-down."

A recent wetting layer model was developed for the case of relatively active substrates. Specifically for $\mathrm{D}_{2} \mathrm{O} / \mathrm{Ru}(0001)$, a partially dissociated wetting layer has been proposed, in which half the $\mathrm{D}_{2} \mathrm{O}$ molecules dissociate to yield an adsorbed, $\mathrm{D}_{2} \mathrm{O}+\mathrm{OD}$ network [3-5]. This structure explains the only low energy electron diffraction (LEED) structure determination for a water overlayer [6], underlining the idea that new quantitative experimental data and their theoretical analysis is likely to yield novel microscopic insight into wetting.
PACS numbers: 68.43.Bc, 68.37.Ef, 68.43.Fg, 82.65.+r
With this in mind, scanning tunneling microscopy (STM) images, acquired near half monolayer coverage of $\mathrm{D}_{2} \mathrm{O}$ on $\mathrm{Pd}(111)$ and at $100 \mathrm{~K}$, are intriguing [7]. Here, we explore the structures of these overlayers using density functional theory (DFT) and STM simulations, and show that none of the existing models of water adsorption can explain the most striking experimental observations. Instead, the overlayers are comprised mostly of planar hexamer rings of nearly flat-lying water molecules. On this basis we set forth a new "2D-water rule," yielding a natural account of the patterns seen in the STM images.

Details of the experiments interpreted herein are reported in Ref. [8], a paper exploring how water monomers adsorb at $40 \mathrm{~K}$, and how they diffuse and aggregate to form small water clusters. Examples of the large water clusters considered here, acquired at $100 \mathrm{~K}$, are shown in Fig. 1(a). They include long chains of hexagonal units whose width never exceeds a few hexagonal cells. A lace structure often forms [Fig. 1(b)], where the hexagonal units combine into extended and narrow chains, with vacant adsorption sites or holes in a periodic network.
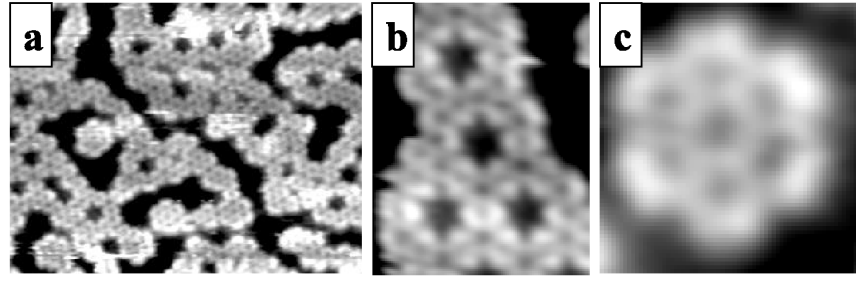

FIG. 1. (a) $(175 \times 135 \AA)$ STM image of $\mathrm{D}_{2} \mathrm{O}$ clusters on $\operatorname{Pd}(111)$ at $100 \mathrm{~K}$. (b) $(41 \times 53 \AA)$ zoom-in image of the lace structure clearly showing star-shaped defected areas (dark in STM). (c) $(20 \times 20 \AA$ Å zoom-in image of the rosette structure. With scanning parameters set to $200 \mathrm{pA}$ tunnel current and $-100 \mathrm{mV}$ bias voltage, no tip influence was detected. 
One also commonly sees seven connected hexagons arranged in a "rosette" [Fig. 1(c)]. All observed clusters display a honeycomb contrast, internally, with $(\sqrt{3} \times$ $\sqrt{3}) R 30^{\circ}$ hexagonal symmetry, and enhanced corrugation at the cluster edges. Depositing water to higher coverage yields second-layer molecules, as does an anneal cycle to $130 \mathrm{~K}$. Thus, these structures are metastable and their formation is governed by kinetic accessibility, not thermodynamics [7], unlike the much discussed periodic water layer formed on $\mathrm{Ru}(0001)$ by a $150 \mathrm{~K}$ anneal [6].

To interpret the experimental images, we have performed STM image simulations for representative structures found, via DFT optimization, to be metastable. This combined DFT/STM simulation approach is particularly useful in the present case because DFT predicts several water cluster structures on $\operatorname{Pd}(111)$ with nearly equal binding energies. The DFT calculations were performed in periodic supercells within the plane-wave pseudopotential formalism. Vanderbilt ultrasoft pseudopotentials [9] and the Perdew-Wang '91 generalized gradient approximation were used [10]. Calculations were performed on 3 to 5 layer Pd slabs in a variety of supercells, ranging from small $(\sqrt{3} \times \sqrt{3}) R 30^{\circ}$ cells to $p(6 \times 6)$ cells. Two DFT codes, VASP [11] and CASTEP [12] were used, which yielded very similar results for cluster geometries.

STM image simulations were performed with the GREEN code [13], whereby the STM current is evaluated within a one-electron elastic scattering approximation after calculating the system's Green's function by matching the surface and tip apex up to first order. The electronic structure is calculated within the extended Hückel theory (EHT) employing the parametrization scheme of Ref. [14]. In particular, we exercised special care in choosing the EHT parameters for the species at the sample surface. Herein we show results obtained with a sharp tip modeled by an isolated 5-atom $\mathrm{Pt}$ pyramid stacked on top of a $\mathrm{Pt}(100)$ surface [13]. Cross checking with other tip models, we found that those with a sharp topography at the apex provided very similar images, while blunter tips [e.g., W(111)] yielded more distorted images that were not seen by experiment. Test calculations for a flat-lying $\mathrm{H}_{2} \mathrm{O}$ molecule on $\mathrm{Pd}(111)$ (most favorable configuration predicted by DFT [15]) imaged with sharp tips quantitatively reproduced the STM round bump ( $0.7 \AA$ ) observed for $\mathrm{H}_{2} \mathrm{O}$ monomers [8].

The central issue in trying to understand water clusters on $\mathrm{Pd}(111)$ is to identify where the $\mathrm{H}$ or $\mathrm{D}$ atoms reside, since they cannot be resolved in the images. Are the lace and rosette structures constituted of icelike, H-up, or Hdown clusters? Or, possibly, are some of the water molecules dissociated? Figure 2(a) shows a generic overlayer model for $0.5 \mathrm{ML}$ (monolayer) water coverage. The figure illustrates a $p(6 \times 6)$ unit cell containing 9 flat water molecules (white circles) and another 9 species (gray circles), which may be up or down water molecules or $\mathrm{O}-\mathrm{H}$ fragments. This structure embodies the three existing models for extended water overlayer adsorption, but with six molecules removed from the unit cell so as to leave a periodic pattern that closely resembles the experimental lace structure of Fig. 1(b). However, the ability of existing models such as these to explain the overlayer structures observed here can be questioned. First, they offer no explanation for the fixed width of the chains of the lace structure and the unique diameter of the rosettes. Second, the species at the lace's ring edges alternate between flat (white) and nonflat (gray) molecules. Such alternation should impart threefold symmetry on the contrast of the vacant regions. What is observed, however, is sixfold starlike symmetry, apparently ruling these models out. Hopes of using DFT energy minimization to eliminate candidate rosette and lace structural models are quickly dashed. Calculations, performed for up, down, and half-dissociated lace models, do show that all three configurations are metastable; that is, they do not spontaneously undergo drastic structural changes relative to the ideal $(\sqrt{3} \times \sqrt{3}) R 30^{\circ}$ nondefected overlayer geometries. Nonetheless, the adsorption energies
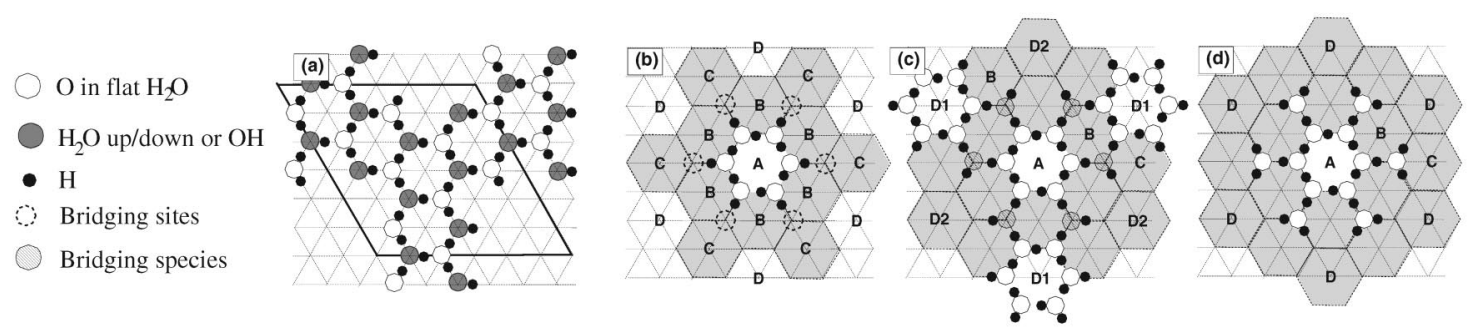

FIG. 2. (a) Illustration of lacelike $p(6 \times 6)$ superstructures on Pd(111), built by removing molecules from icelike bilayers. Pd surface atoms are at the crossings of the thin lines. (b)-(d) Illustration of the mechanism by which planar hexamers bind to each other. In (b) the gray filled hexagons define the "exclusion zone" where another $A$ type hexamer cannot adsorb. (c) Scheme for the lacelike structure constructed after placing tilted species (hatched circles) at the bridging sites. The arrangement of the bridging species extends the exclusion zone to the $D 2$ sites. (d) Scheme for the rosette type structure after placing flat-lying water molecules at the bridging sites. Now, the exclusion zone extends to all $D$ sites. See text for further details. 
TABLE I. DFT binding energies of Ice $\mathrm{I} h$ [3] and various adstructures, per deposited $\mathrm{H}_{2} \mathrm{O}$. Zero-point energy not included, would bring the dissociated and intact molecule energies closer, because it favors rupturing stiff O-H bonds [4].

\begin{tabular}{lc}
\hline \hline Model (arrangement) & $E_{\mathrm{ads}}\left[\mathrm{eV} / \mathrm{H}_{2} \mathrm{O}\right]$ \\
\hline Ice I $h$ (bulk) & 0.71 \\
H-up (complete bilayer) & 0.50 \\
H-down (complete bilayer) & 0.56 \\
Dissociated (complete bilayer) & 0.46 \\
H-up (Lace) & 0.52 \\
H-down (Lace) & 0.54 \\
Dissociated (Lace) & 0.51 \\
Flat + edge H-down (lace) & 0.56 \\
Flat + edge dissociated (lace) & 0.53 \\
Flat + edge H-down (rosette) & 0.56 \\
Flat + edge dissociated (rosette) & 0.51 \\
\hline \hline
\end{tabular}

given in Table I show that the defect structures are no more stable than their ideal $(\sqrt{3} \times \sqrt{3}) R 30^{\circ}$ counterparts, and still less against formation of bulk ice $\mathrm{I} h$ [3].

We therefore explored alternate clustering mechanisms, based on the knowledge that isolated $\mathrm{H}_{2} \mathrm{O}$ monomers preferentially adsorb on close-packed metal surfaces with both O-H bonds nearly parallel to the surface [15] and additional DFT optimizations that identified several relatively stable adsorbed $\mathrm{H}_{2} \mathrm{O}$ hexamers comprising mostly flat-lying $\mathrm{H}_{2} \mathrm{O}$ molecules. An example of one such hexamer, composed of six nearly flat $\mathrm{H}_{2} \mathrm{O}$ molecules [16], is shown in Fig. 2(b).

Proceeding from the ansatz that a flat hexamer is the basic building block during low- $T$ water clustering, different hexamer aggregates may be generated by invoking the following rule for 2D water growth: Each water molecule prefers to lie approximately parallel to the surface, forming $\mathrm{H}$ bonds such that $\mathrm{O}-\mathrm{O}-\mathrm{O}$ angles are about $120^{\circ}$. This $2 \mathrm{D}$-water rule may be considered as an alternative to the traditional ice rule [1] (and also to one of Doering and Madey's surface modified ice rules [17]), that requires oxygens to be in a tetrahedral environment. However, since each molecule donates $2 \mathrm{H}$ bonds but accepts only one, there is an imbalance between donors and acceptors and defect-free networks of flat molecules can never be achieved. Instead defects are necessary, which leads to the lace and rosette structures observed.

Let us now consider in detail how this rule leads to the observed lace and rosette patterns. As depicted in Fig. 2(b), one may define an exclusion zone around each flat $A$ hexamer (shaded region in the figure) within which another similar flat hexamer cannot adsorb without violating the 2D-water rule. The exclusion zone covers the first $(B)$ and second $(C)$ nearest neighbor sites, leaving sites $D$ as the closest ones where another flat hexamer may adsorb. The key problem, then, is to identify the bonding mechanism between a flat hexamer at $A$ and another at $D$.
The obvious choice is to place a nonflat water molecule at the "bridging site," with one O-H-bond parallel to the surface and the other dangling, with the $\mathrm{H}$ either up or down or missing, in order to respect the 3D ice rules. This is depicted in Fig. 2(c), where the six available bridging sites are occupied. Notice that this arrangement facilitates growth along three symmetry equivalent directions $[A-D 1$ in Fig. 2(c)], but prevents growth along the other three $[A-D 2]$. This way, the chainlike geometry, and fixed chain width, characteristic of the lace-type structure are naturally derived. In fact, the structure corresponds to a 2D periodic tiling arising from the combination of flat hexamers and bridging species.

On the other hand, if flat molecules are placed at the bridging sites [Fig. 2(d)], then the growth is inhibited along all six directions, since there is an $\mathrm{O}-\mathrm{H}$-bond pointing to all potential $D$ hexamers, extending the exclusion zone. In this case, the nucleus of a rosette structure is left. The rosette is completed by closing each of the six $B$ hexamers with two water molecules, suitably oriented to respect the 3D ice rules (i.e., with at least one of the two nonflat). Thus, all the structures experimentally imaged may now be easily generated by combining flat and/or nonflat molecules at the bridging sites, the former inhibiting the growth while the latter facilitating it.

We now verify this novel growth model and address the nature of the bridging and edge species in the lace and rosette structures. DFT calculations for these structures were performed in the same $p(6 \times 6)$ unit cell used for the bilayer models. For the lace model, structure optimi-
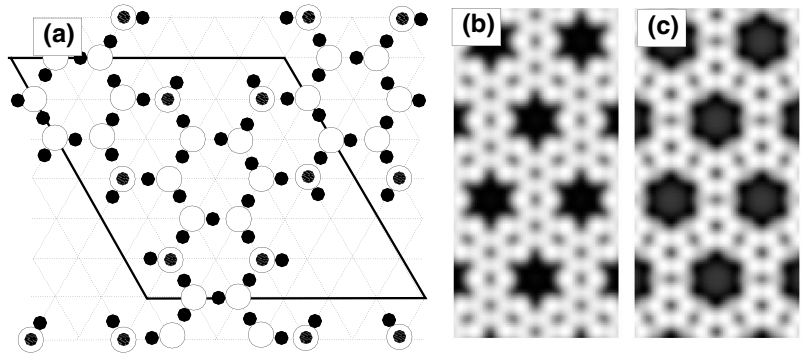

(d)
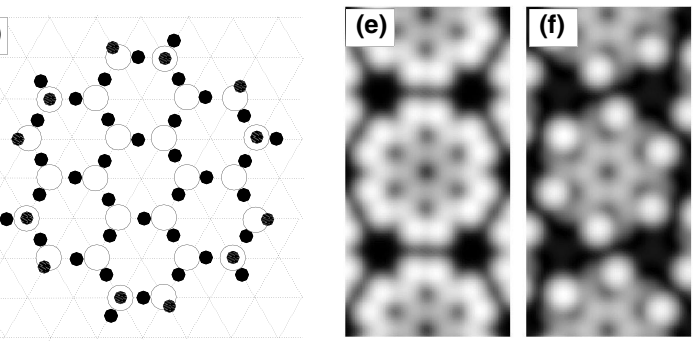

FIG. 3. (a) The optimized lace structure. Small gray circles are $\mathrm{H}$ atoms below the $\mathrm{O}$ atoms (down configuration). The remaining atoms are labeled as in Fig. 2. (b) STM topographic image simulation for this model (with $I=0.1 \mathrm{nA}$ and $V=$ $100 \mathrm{mV}$ ). (c) Same as (b) but for a model where the bridging molecules are $\mathrm{O}-\mathrm{H}$ species. (d)-(f) Same as (a)-(c) but for the rosette structure. 
zations reveal relatively stable phases for both $\mathrm{H}$-down or hydroxyl bridging species. However, with adsorption energies of 0.56 and $0.53 \mathrm{eV} / \mathrm{H}_{2} \mathrm{O}$ compared to $0.71 \mathrm{eV}$ for bulk ice $\mathrm{I} h$ (see Table I), it is clear that reasoning on the basis of maximal binding energy cannot help us judge which metastable structure is represented by the STM images. On the other hand, direct simulation of the images based on the DFT structures offers unambiguous evidence that the bridging species is a water molecule in an H-down configuration. The model and its associated STM simulated image are presented in Figs. 3(a) and $3(\mathrm{~b})$; the nice agreement with the experimental image of Fig. 1(b) is evident. Overall corrugations are $0.6 \AA$, in good accord with the STM data. The out-of-ring O-H bonds of the bridging water molecules protrude into the vacancy region, thus generating the characteristic star shape, while the bridging $\mathrm{O}$ atoms appear only slightly brighter than those belonging to flat molecules, despite the fact that they are $0.5 \AA$ farther away from the surface. STM images simulated when the bridging species are O$\mathrm{H}$ molecules, shown in Fig. 3(c), are at variance with the experimental images since the oxygens in the hydroxyls yield a markedly smaller tunneling signal (less bright).

For the rosettes shown in Fig. 2(d), DFT calculations were performed assuming one edge molecule in each of the $B$ hexamers to be initially flat and the other one either in the H-down configuration or half-dissociated. The STM simulations leave no doubt that the edge species are intact, H-down molecules. The model and the theoretical images are shown in Fig. 3(d)-3(f). The agreement is good for the H-down configuration [Fig. 3(e)], correctly reproducing the brighter contrast at the edges of the cluster. The overall corrugation is $0.7 \AA$, again within the experimental range. This time the $\mathrm{O}$ atoms of the edge molecules are $0.7 \AA$ farther from the surface than those of the internal, flat hexamers. In fact, the structure optimization reveals that each of the initially flat edge water molecules adopts pseudo $\mathrm{H}$-down configurations, with its dangling $\mathrm{H}$-bond lifting the $\mathrm{O}$ atom to almost the same height as that of the other H-down edge molecules [see Fig. 3(d)]. On the contrary, for the $\mathrm{O}-\mathrm{H}$ configuration [Fig. 3(f)], the dissociated molecules appear less bright, ruling this model out.

In summary, water adsorbs on $\mathrm{Pd}(111)$ in the submonolayer regime with a novel structure, in which all water molecules remain intact yet the majority lie parallel to the surface. This overlayer-model is not based on the puckered hexagonal structure of ice $\mathrm{I} h$, nor on any recent variations such as the H-down, or "dissociated" ice models. Instead, we find an arrangement only possible in the submonolayer regime, wherein most of the molecules lie flat against the surface. Central to the proposed systematization of water cluster morphologies, depicted in
Figs. 2(b) $-2(d)$, are symmetric planar hexamer building blocks, and the specific nature of the molecules that connect them. We have shown how, depending on the nature of the bridging molecules (H-down or flat) the characteristic lace or rosette structures evolve as two highly symmetric outcomes. Total energy calculations coupled to STM simulations fully confirm this novel overlayer growth, and lead to the proposal of a new rule to generate pictures of the kinetically accessed water structures seen. How important flat water arrangements may be to other water-metal systems remains to be seen.

M. L. B. and J.C. thank the European COST Action No. d26-0005-02. J. C.'s work was supported by the Spanish DGICyT under Contract No. MAT2001-1956. A. M. thanks Gonville and Caius College, Cambridge for support. P. J. F. thanks the Chemistry Department of Cambridge University and Columbia University's Center for Integrated Science \& Engineering for their hospitality. P. J. F.'s work and that of M. S. and the L. B. L. team were supported by the U.S. Department of Energy, Office of Basic Energy Sciences, Division of Material Sciences. Sandia is operated for the U.S. DOE under Contract No. DE-AC04-94AL85000. L. B. L. is supported under Contract No. DE-AC03-76F00098.

*Electronic address: mbocquet@ens-lyon.fr

[1] M. A. Henderson, Surf. Sci. Rep. 46, 1 (2002); P. A. Thiel and T. E. Madey, Surf. Sci. Rep. 7, 211 (1987).

[2] H. Ogasawara et al., Phys. Rev. Lett. 89, 276102 (2002); S. Meng et al., Phys. Rev. Lett. 89, 176104 (2002); A. Michaelides, A. Alavi, and D. A. King, Phys. Rev. B 69, 113404 (2004).

[3] P. J. Feibelman, Science 295, 99 (2002).

[4] P. J Feibelman, Phys. Rev. B 67, 035420 (2003).

[5] A. Michaelides, A. Alavi, and D. A. King, J. Am. Chem. Soc. 125, 2746 (2003).

[6] G. Held and D. Menzel, Surf. Sci. 316, 92 (1994).

[7] T. Mitsui et al. (to be published).

[8] T. Mitsui et al., Science 297, 1850 (2002).

[9] D. Vanderbilt, Phys. Rev. B 41, 7892(R) (1990).

[10] J. P. Perdew et al., Phys. Rev. B 46, 6671 (1992).

[11] G. Kresse and J. Furthmuller, J. Comput. Mater. Sci. 6, 15 (1996); Phys. Rev. B 54, 11169 (1996).

[12] CASTEP 4.2 Academic Version, licensed under the UKCPMSI Agreement, 1999; M. C. Payne et al., Rev. Mod. Phys. 64, 1045 (1992).

[13] J. Cerdá et al., Phys. Rev. B 56, 15885 (1997); http:// www.icmm.csic.es/jcerda/

[14] J. Cerdá and F. Soria, Phys. Rev. B 61, 7965 (2000).

[15] A. Michaelides et al., Phys. Rev. Lett. 90, 216102 (2003).

[16] The adsorption energy per $\mathrm{H}_{2} \mathrm{O}$ in this isolated hexamer is $0.56 \mathrm{eV}$.

[17] D. L. Doering and T. E. Madey, Surf. Sci. 123, 305 (1982). 